\title{
Eongenital Short Ligament of Treitz Presenting as Superior Mesenteric Artery Syndrome
}

\section{Tony Joseph, Prasanth Sobhan, Suthanu Bahuleyan, George Peter, Srijaya Sreesh,} Premalatha Narayanan, Kattoor Ramakrishnan Vinayakumar

\section{Department of Medical Gastroenterology, Government Medical College,}

Thiruvananthapuram, Kerala, India.

\section{Abstract:}

Superior mesenteric artery (SMA) syndrome occurs due to compression of the third portion of duodenum as it crosses anterior to aorta at the level of $L 3$ vertebral body. We report a case of 40 year old female, who presented with recurrent bilious vomiting of 3 months duration and significant weight loss. Clinical examination revealed features of proximal small bowel obstruction. Contrast enhanced computed tomography showed reversed SMA-SMV axis and encircling of the proximal jejunal loops surrounding the superior mesenteric artery with possible diagnosis of malrotation with intermittent volvulus. However, barium meal follow through with corroborative ultrasound Doppler revealed features suggestive of superior mesenteric artery syndrome. Intraoperatively, a short thick ligament of Treitz was found with no features of malrotation. The ligament of Treitz was released, roux-en-Y duodenojejunal bypass surgery was done.

Key words: Superior Mesenteric Artery, Intestinal Volvulus, Duodenum, Roux-en-Y Anastomosis, Vomiting.

\section{Introduction}

Superior mesenteric artery syndrome occurs due to narrowing of the space between origin of superior mesenteric artery from aorta at the level of L3 vertebral body causing compression of the third portion of duodenum. Apart from a case report about its description in utero, it has been reported usually as a syndrome of adulthood that resulted from unintentional weight loss due to various acquired causes.

We report a case of 40 year old female, who presented to us with recurrent bilious vomiting of 3 months duration and significant weight loss of
$12 \mathrm{~kg}$ who was diagnosed as superior mesenteric artery syndrome after radiological evaluation, but later turned out to be the result of short thick ligament of Treitz intraoperatively. We believe this is the first case report of congenital short ligament of Treitz presenting as superior mesenteric syndrome in an adult.

\section{Case Report}

40 year old female presented to our department with 3 months history of recurrent bilious vomiting. Episodes were preceded by upper abdominal

Corresponding Author: Dr. Tony Joseph

Email: drtony.gastro@gmail.com

Received: February 24, 2015 | Accepted: June 23, 2015 | Published Online: July 20, 2015

This is an Open Access article distributed under the terms of the Creative Commons Attribution License (creativecommons.org/licenses/by/3.0)

Conflict of interest: None declared | Source of funding: Nil | DOl: http://dx.doi.org/10.17659/01.2015.0080 
pain and fullness, and relief of symptoms following vomiting. She also noted relief of symptoms following adoption of recumbent posture postprandially. Initially vomiting was 2-3 episodes per day, but had worsened to 6-8 episodes per day recently. She also had poor appetite since one month. There was no history of stale food vomitus and no significant past medical or surgical history.

On clinical examination there was a weight loss of $20 \mathrm{~kg}$. Abdominal examination showed upper abdominal fullness and hyperperistaltic bowel sounds with no succession splash. Her laboratory parameters were normal. Differential diagnosis considered were proximal duodenal obstruction, with possible etiologies like tuberculosis, Crohns disease and adenocarcinoma. Other possibilities thought of were annular pancreas, midgut malrotation and paraduodenal hernia.

Investigations showed haemoglobin of $10.2 \mathrm{~g} / \mathrm{dL}$, among liver function tests albumin was $2.8 \mathrm{~g} / \mathrm{dL}$. Rest of the investigations including potential markers of malignancy were normal. Upper endoscopy revealed stasis of food in antrum, scope was negotiated into first part of duodenum without difficulty and there was dilation upto proximal portion of third part of duodenum.

Contrast enhanced computed tomography (CECT) showed dilated stomach and proximal duodenum with the transverse portion of the duodenum seen crossing to the left side. The visible parts of the large bowel showed normal lumen and walls. Caecum was seen in the right iliac fossa. The superior mesenteric vein was positioned to the left of superior mesenteric artery (reversed SMASMV axis). Encircling of the proximal jejunal loops surrounding the superior mesenteric artery was also noted (Whirl sign). There was fat stranding of the mesentery suggestive of mesenteric congestion and also clustering of jejunal loops towards left hypochondrium. The imaging features with background history suggested malrotation with intermittent volvulus [Fig.1].

Radiological study with barium meal follow through demonstrated dilated first and second part of duodenum and narrow short segment of middle $1 / 3^{\text {rd }}$ of third part of duodenum [Fig.2]. Corroborative ultrasound Doppler sonography confirmed reduced aortomesenteric angle $8.2^{\circ}$ and aortomesenteric distance $3.8 \mathrm{~mm}$. The findings were suggestive of superior mesenteric artery syndrome.

Patient was taken up for surgery. Intraoperatively dilated first, second and third portion of duodenum was found suggestive of SMA syndrome. Duodeno-jejunal flexure was found high up behind the stomach and a short thick band originating from it was noted passing around the origin of celiac artery and superior mesenteric artery was also noted [Fig.3]. No separate ligament of Treitz was found with caecum in normal position. Band was released and Roux-en-Y duodenojejunal bypass surgery was done. She was put on total

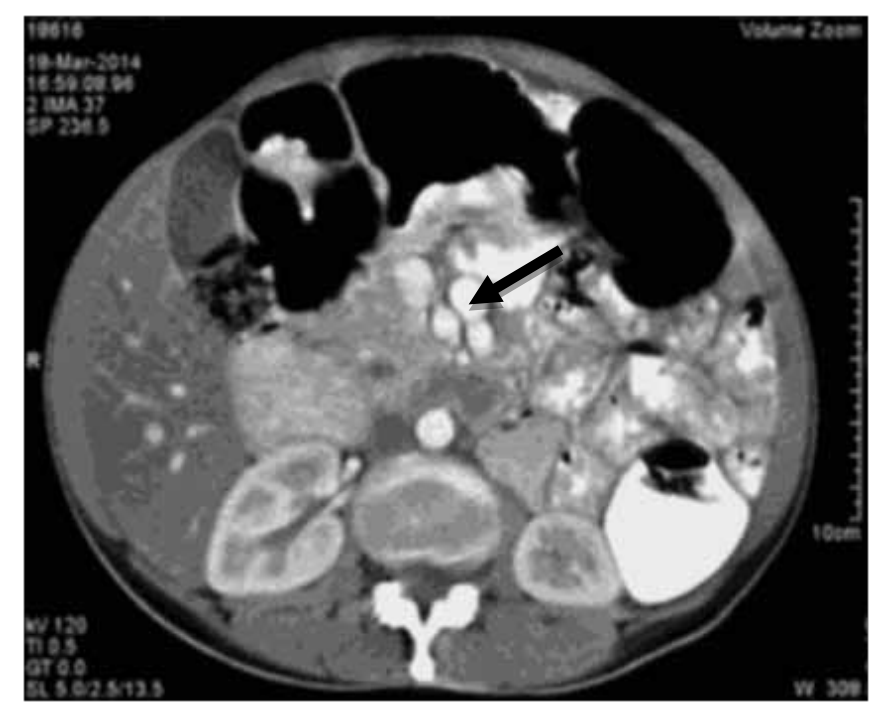

Fig.1: Stomach and proximal duodenum is dilated. Note the reversed SMA-SMV axis (arrow), proximal jejunal loops surrounding SMA producing "whirl" sign. 
parenteral nutrition for 3 weeks. Postoperatively patient had a prolonged recovery over one month because of severe weight loss and malnutrition. On six months follow up, she attained gradual weight gain of $12 \mathrm{~kg}$.

\section{Discussion}

Superior mesenteric artery syndrome was first reported by Rokitanski in 1861, and details were described by Wilkie in his largest case series in 1927, hence it is also known as Wilkie's syndrome. The third portion of duodenum crosses anterior to aorta at the level of L3 vertebral body. Superior mesenteric artery arises from anterior aspect abdominal aorta at an acute angle at the level of $L 1$ vertebral body and extends caudally into mesentery. It is enveloped in fatty and lymphatic tissue. Superior mesenteric artery syndrome occurs primarily due to loss of intervening mesenteric fat, causing narrowing of space between the aorta and superior mesenteric artery leading to compression of third part of duodenum $[1,2]$. Normal aortomesenteric angle range from $25^{\circ}$ to $60^{\circ}$ and aortomesenteric distance 10 to $28 \mathrm{~mm}$ [3]. The angle was found to correlate with body mass index (BMI) in a study [4]. Any condition that leads to prolonged bed rest, most commonly severe debilitating illnesses can predispose to superior mesenteric artery syndrome. Weight loss may not responsible in all cases. Anatomical abnormalities, both congenital and acquired can also contribute. Anatomical alternations such as following corrective surgery for scoliosis (hence the name "cast syndrome"), shortening of esophagus after esophagectomy can also narrow the aortomesenteric angle $[5,6]$.

Congenital short ligament of Treitz producing abnormal cephalad position of superior mesenteric artery has been reported in identical twins. There is also a case report of superior mesenteric artery

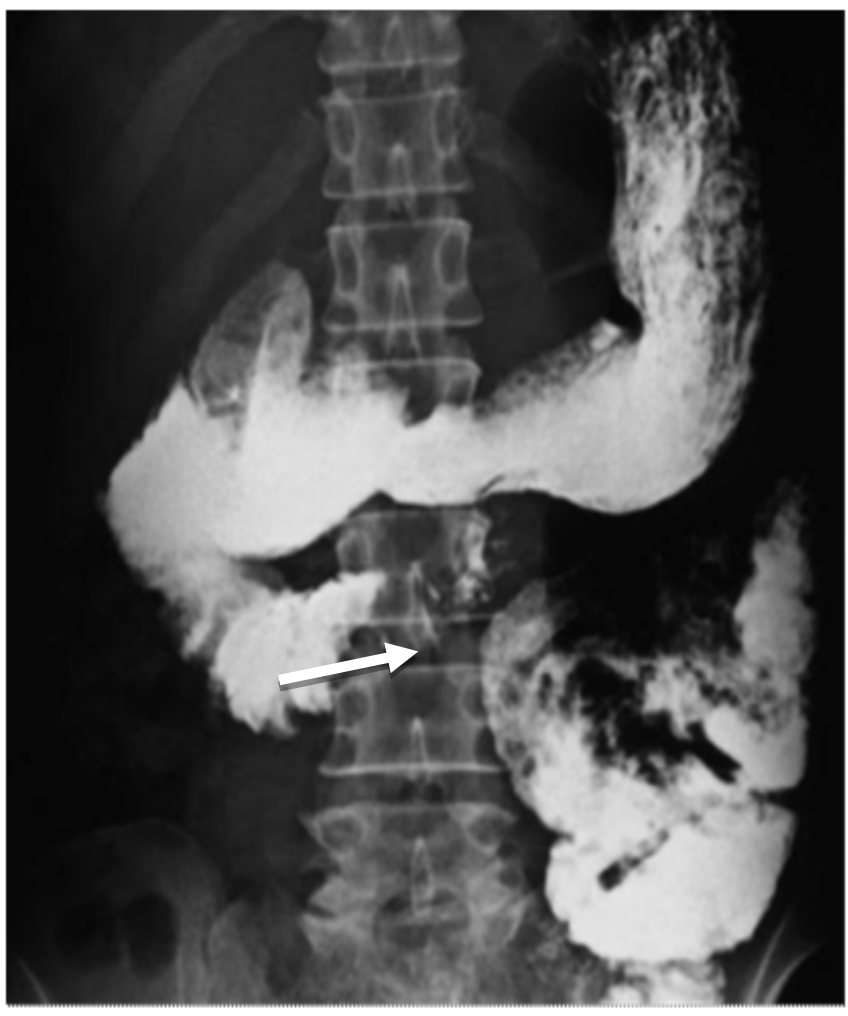

Fig.2: Barium meal follow through - note that at $120 \mathrm{~min}$, dilated proximal duodenum with persistent narrowing of middle third of third part of duodenum (arrow) suggestive of superior mesenteric artery syndrome.

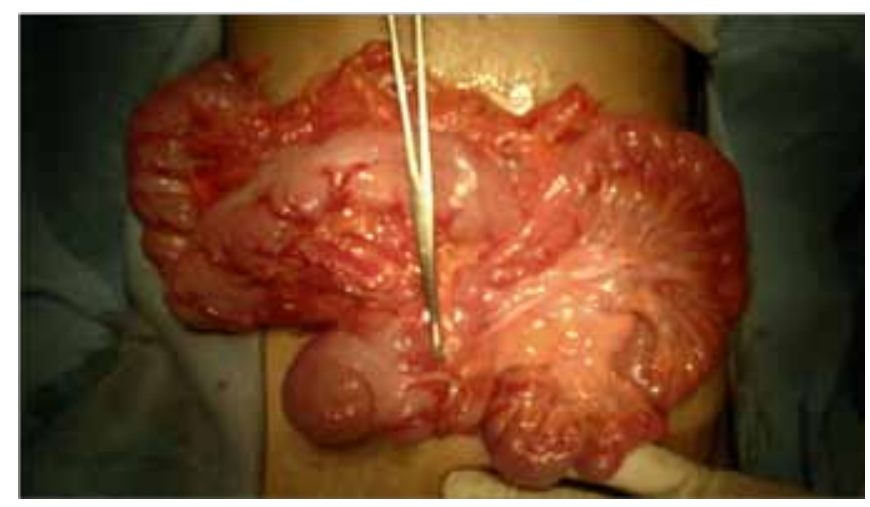

Fig.3: Intraoperative picture - Forceps point to short thick ligament of treitz running between duodeno-jejunal flexure and origin of SMA. 
syndrome in utero $[7,8]$. A low origin of superior mesenteric artery congenitally can also produce similar picture.

We believe that in this patient, the short thick ligament of Treitz predisposed to some form of partial malrotation or intermittent volvulus that had caused recurrent episodes of abdominal pain and bilious vomiting. Intermittent symptoms might had caused poor appetite leading to weight loss. Doppler ultrasonography of abdomen showed reduced aortomesenteric angle $8.2^{\circ}$ and aortomesenteric distance $3.8 \mathrm{~mm}$ in our patient confirming the diagnosis of superior mesenteric artery syndrome.

Asymptomatic postoperative course and satisfactory weight gain on follow up also holds our hypothesis true.

\section{Conclusion}

Superior mesenteric artery syndrome is usually described as acquired syndrome of adulthood due to various causes leading to unintentional weight loss. Our case report presents an unusual coexistence of a congenital short ligament of Treitz in an adult causing superior mesenteric artery syndrome. The persistence of such rare anatomical abnormalities should be always looked for while evaluating cases recurrent bilious vomiting and weight loss, if etiologies such as malrotation cannot be convincingly ruled out.

\section{References}

1. Rokitanski CV. Vienna. $3^{\text {rd }}$ edn. Vol. 3. Braumulller and Siedel: 1861. Lehrbuch der Pathologische Anatomie; pp. 187.

2. Wilkie D. Chronic Duodenal ileus. Am J Med Sci. 1927;173:643-649.

3. Applegate GR, Cohen AJ. Dynamic CT in superior mesenteric artery syndrome. J Comput Assist Tomogr. 1988; 12:976-980.

4. Ozkurt H, Cenker MM, Bas N, Erturk SM, Basak $M$. Measurement of the distance and angle between the aorta and superior mesenteric artery: Normal values in different BMI categories. Surg Radiol Anat. 2007;29:595.

5. Crowther MA, Webb PJ, Eyre-Brook IA. Superior mesenteric artery syndrome following surgery for scoliosis. Spine (Phila Pa 1976) 2002;27:E528.

6. Cho KR, Jo WM. Superior mesenteric artery syndrome after esophagectomy with cervical esophagogastrostomy. Ann Thoracic Surg. 2006;82:e37.

7. Iwaoka Y, Yamada M, Takehira Y, Hanajima K, Nakamura T, Murohisa G, Hirai R, Kitagawa M. Superior mesenteric artery syndrome in indentical twin brothers. Intern Med. $2001 ; 40: 713$.

8. Caspi B, Deutsch H, Grunshpan M, Flidel O, Hagay Z, Appelman Z. Prenatal manifestation of superior mesenteric artery syndrome. Prenat Diagn. 2003;23:932. 